\title{
Mosaic disorders and the Taxonomy of Human Disease
}

\author{
Leslie G. Biesecker, MD
}

In this issue of Genetics in Medicine, Rodriguez-Laguna et al. ${ }^{1}$ identify somatic mosaic variants in PIK3CA in patients who had been assigned the diagnosis of CLAPO syndrome (capillary malformation of the lower lip, lymphatic malformation predominant on the face and neck, asymmetry, and partial/generalized overgrowth; MIM 613089). The findings in this paper raise interesting and important questions, the first being the question of whether CLAPO syndrome was a legitimate diagnostic entity to begin with.

The term "CLAPO syndrome" was coined in a 2008 publication by the research group, which also wrote the accompanying paper in this issue of the Journal. They described six patients with what they proposed to be a distinct clinical diagnostic entity. ${ }^{2}$ This entity was discussed in several review articles, ${ }^{3-5}$ which included several of the original report authors but also others who are authoritative in this field, and were subject to peer and/or editorial evaluation. None of the papers that followed the original report suggested that CLAPO was not a valid diagnostic entity. Furthermore, CLAPO is a phenotype entry in OMIM. I conclude that prior to the findings published in this issue, CLAPO was a valid clinical diagnostic entity, to the extent that that phenotypic classification alone is a valid approach to disease taxonomy (which is a critical caveat).

Based on the findings by Rodriguez-Laguna et al., ${ }^{1}$ we now know that CLAPO is associated with mosaic-activating variants in $P I K 3 C A$, raising a new question of the validity of this diagnostic entity. Mosaic PIK3CA variants were first described in a series of four papers in 2012, in patients with a range of phenotypes that included overgrowth, vascular malformation, central nervous system anomalies, and pigmentary findings. Previous descriptors of some of these phenotypes included CLOVES syndrome, megalencephalycapillary malformation (MCAP or M-CM), KlippelTrenaunay syndrome, and isolated anomalies such as singledigit macrodactyly. This litany of associations with mosaic PIK3CA variants was surprising and confusing. The CLAPO findings add to that conundrum, and the situation begs for rationalization and clarity. In 2014 we gathered a group of experts at the National Institutes of Health to wrestle with this challenge and moved toward a unification of the distinct entities under the umbrella term of PIK3CA-related overgrowth spectrum (PROS). ${ }^{6}$ The designation of this as a spectrum acknowledged a critical fact: no rational, objective, and clear clinical delineation can be made among these entities. Since our designation of the CLOVE syndrome acronym (for congenital, lipomatous overgrowth, with vascular anomalies and epidermal nevi; later amended to CLOVES to add skeletal findings ${ }^{7}$ ) in 2009, we have come to recognize that this "syndrome" was an artifact of the clinical pattern-recognition approach to disease delineation. It was correct that the patients we diagnosed with CLOVES were distinct from those with Proteus syndrome (as subsequently confirmed by molecular data ${ }^{8}$ ), but it was incorrect that those five findings represented a distinct clinicopathologic entity. Indeed, we have come to recognize just as many, or more, patients with four, three, two, or only one of those findings, all of which are due to the same or similar variants in PIK3CA. These variant phenotypes blend continuously with one another. This recognition led us to conclude that there is no rational, objective, definable way to distinguish, for example, a patient with CLOVES syndrome from many other patients with PIK3CA-related overgrowth, and therefore that CLOVES syndrome does not exist as a valid, unitary, clinical diagnostic entity. The same is probably true for Klippel-Trenaunay syndrome, hemihyperplasia-multiple lipomatosis, CLAPO, and all other mosaic overgrowth disorders associated with PIK3CA-activating variants. While the designation of CLAPO as a distinct clinical entity was reasonable in 2008, with the availability of precise molecular data this designation is not supportable today. The use of CLAPO as a clinical diagnostic entity should be discontinued, and patients with that diagnosis should instead be rediagnosed as having PROS.

These considerations of PROS have implications far beyond patients with somatic variants in PIK3CA. The question is whether diseases should be taxonomically organized according to their molecular or their phenotypic attributes. Our current diagnostic thinking is overwhelmingly phenotypic. But, as molecular insights accrue, a tidal wave of evidence in favor of molecular taxonomy is building. In somatic cancer genetics, it is already here. Oncologists are beginning to treat tumors based on their molecular profile, irrespective of their clinical or histologic attributes. But cancer provides important evidence against a unitary molecular taxonomy of

${ }^{1}$ Medical Genomics and Metabolic Genetics Branch, National Human Genome Research Institute, National Institutes of Health, Bethesda, Maryland, USA. Correspondence: Leslie G. Biesecker (lesb@mail.nih.gov)

Submitted 18 October 2017; accepted 25 October 2017; advance online publication 7 December 2017. doi:10.1038/gim.2017.213 
PIK3CA-related phenotypes. Many tumors have somatic PIK3CA variants, many of which are exactly the same somatic variants as those found in patients with PROS. Such a PIK3CA variant in a malignancy is in a context of dozens or hundreds of other somatic variants. Even if a clinician is willing to accept PROS as a single-spectrum disorder, he or she would be very unlikely to say that a patient with lung cancer and PIK3CA variant has the same taxonomic disease entity as that of a patient with isolated macrodactyly. This was indeed the rationale for a multiaxis diagnostic system for disease, ${ }^{9}$ which conceptualized a disease entity as having three primary attributes: phenotype, genotype, and environment. A unitary clinicopathologic disease entity was one that had commonality of each of these three attributes. Clearly, this was not an idea that caught fire, but it has influenced thinking along these lines. Bonnie Pagon, editor of GeneReviews, has adopted a variation of this concept by describing disorders as phenotype-gene dyads. For example, one can describe a patient's diagnosis as being "KRAS-related Noonan syndrome." This is simpler than the multiaxis concept but captures the same fundamental concept. In this framework, KRAS-related Noonan syndrome is distinct from SOS1related Noonan syndrome, just as it is distinct from KRASrelated Schimmelpenning-Feuerstein-Mims syndrome-but distinct in a very different way: in one case phenotypic and another molecular.

It is unjustifiable at this stage to suggest that either the multiaxis or the GeneReviews dyad approach to designating disorders addresses all of the challenges. One challenge is modifiers and complex genetic disorders-these do not fit well into frameworks that are designed for single-gene disorders. Another is again illuminated by mosaicism. There is no rational lower boundary that can be established for the mosaicism level. Patients with recognizable PROS phenotypes have variant allele frequencies between a few percent and 50\% in the affected parts of the body, but the cells with the variants may be a very small fraction of their total number of body cells. What is the lower limit for this? What is the tissue or cell type that harbors that variant, which must play a key role in determining the presence and nature of the phenotype? Undoubtedly, a person with one cell with a PIK3CAactivating variant does not have PROS. Reductio ad absurdum. Addressing this threshold problem will be a challenge.

These limitations aside, what CLAPO and PROS tell us is that we have to begin thinking about disease entities and diagnoses as having more than one attribute re taxonomic classification. The wrong question is whether we should be using phenotype or genotype, because the answer is that we have to use both, simultaneously, in all patients. Establishing precisely how we do that provides an opportunity for further theoretical work as well as clinical and molecular research. But there is no doubt that genomic characterization challenges our unimodal approach to disease taxonomy, and we must develop an effective way to think about our patients on multiple levels, based on multiple attributes, concurrently.

\section{ACKNOWLEDGMENTS}

The author is supported by the Intramural Research Program of the National Human Genome Research Institute. I am grateful to Teri Manolio for helpful comments on a previous draft of this commentary.

\section{DISCLOSURE}

The author receives royalties from Genentech, is an adviser to Illumina, receives honoraria from Wiley-Blackwell, and received support from the Intramural Research Program of the National Human Genome Research Institute (grants HG200328 11 and HG200388 03).

\section{REFERENCES}

1. Rodriguez-Laguna L, Ibañez K, Gordo G, et al. CLAPO syndrome: identification of mosaic activating PIK3CA mutations and delineation of the natural history and phenotype. Genet Med (in press).

2. Lopez-Gutierrez JC, Lapunzina P. Capillary malformation of the lower lip, lymphatic malformation of the face and neck, asymmetry and partial/ generalized overgrowth (CLAPO): report of six cases of a new syndrome/ association. Am J Med Genet A 2008;146A:2583-2588.

3. Del Pozo J, Gomez-Tellado M, Lopez-Gutierrez JC. Vascular malformations in childhood. Actas Dermosifiliogr 2012;103:661-678.

4. Puttgen KB, Pearl M, Tekes A, Mitchell SE. Update on pediatric extracranial vascular anomalies of the head and neck. Childs Nerv Syst 2010;26:1417-1433.

5. Rozas-Munoz E, Frieden IJ, Roe E, Puig L, Baselga E. Vascular stains: proposal for a clinical classification to improve diagnosis and management. Pediatr Dermatol 2016;33:570-584.

6. Keppler-Noreuil KM, Rios JJ, Parker VE, et al. PIK3CA-related overgrowth spectrum (PROS): diagnostic and testing eligibility criteria, differential diagnosis, and evaluation. Am J Med Genet A 2015;167A:287-295.

7. Alomari Al. Characterization of a distinct syndrome that associates complex truncal overgrowth, vascular, and acral anomalies: a descriptive study of 18 cases of CLOVES syndrome. Clin Dysmorphol 2009;18:1-7.

8. Lindhurst MJ, Sapp JC, Teer JK, et al. A mosaic activating mutation in AKT1 associated with the Proteus syndrome. New Engl J Med 2011;365: 611-619.

9. Robin NH, Biesecker LG. Considerations for a multiaxis nomenclature system for medical genetics. Genet Med 2001;3:290-293. 